\title{
Outcomes of RIP Kinase Signaling During Neuroinvasive Viral Infection
}

\author{
Brian P. Daniels and Andrew Oberst
}

\section{Contents}

1 Introduction

1.1 Viral Infections of the CNS

1.2 Programmed Cell Death During Infection

2 Neuroinvasive Viral Infections

2.1 Coronaviruses

2.2 Japanese Encephalitis Virus

2.3 West Nile Virus.

2.4 Zika Virus

3 Lessons from Noninfectious CNS Pathologies

3.1 Spinal Cord Injury

3.2 Traumatic Brain Injury

3.3 Neurodegeneration

4 Conclusions

References

\begin{abstract}
Neuroinvasive viral diseases are a considerable and growing burden on global public health. Despite this, these infections remain poorly understood, and the molecular mechanisms that govern protective versus pathological neuroinflammatory responses to infection are a matter of intense investigation. Recent evidence suggests that necroptosis, an immunogenic form of programmed cell death, may contribute to the pathogenesis of viral encephalitis. However, the receptor-interacting protein (RIP) kinases that coordinate necroptosis, RIPK1 and
\end{abstract}

B. P. Daniels

Department of Cell Biology and Neuroscience, Rutgers University, Piscataway, NJ 08854, USA

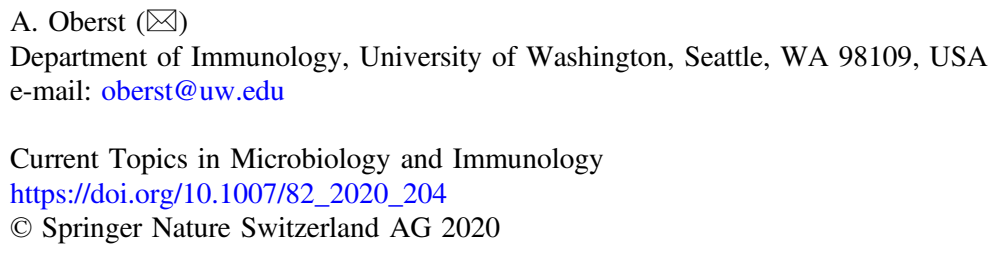


RIPK3, also appear to have unexpected, cell death-independent functions in the central nervous system (CNS) that promote beneficial neuroinflammation during neuroinvasive infection. Here, we review the emerging evidence in this field, with additional discussion of recent work examining roles for RIPK signaling and necroptosis during noninfectious pathologies of the CNS, as these studies provide important additional insight into the potential for specialized neuroimmune functions for the RIP kinases.

\section{Introduction}

\subsection{Viral Infections of the CNS}

Diseases associated with neuroinvasive viral infections are a significant and growing burden on global health. The central nervous system (CNS) is normally protected from infection by physical and biological barriers that limit viral neuroinvasion (Daniels and Klein 2015). Severe neuroinvasive viral infections occur with higher frequency in very young and elderly patients, as well as those who are immunocompromised (Murray et al. 2006; Singh et al. 2015). Genetic factors can also predispose otherwise healthy adult patients to neuroinvasive infection, though the mechanisms that drive susceptibility in these cases are poorly understood (Kallianpur and Levine 2014; Long et al. 2016). Viral infection of the CNS often results in life-threatening pathological outcomes, including encephalitis, meningitis, and neuronal death (Salimi et al. 2016). As the CNS is highly susceptible to injury and limited in its capacity for repair, even those patients who survive viral encephalitis frequently experience long-term sequelae, including emotional dysregulation, altered personality, memory loss, and dementia (Ronca et al. 2016; Patel et al. 2015; Klein et al. 2017).

Beyond infection in the adult CNS, neuroinvasive viral infection in utero is also associated with severe pathological outcomes, including spontaneous abortion, developmental abnormalities, and lifelong intellectual disability (Coyne and Lazear 2016; Neu et al. 2015). Indeed, alarmingly high rates of neonatal microcephaly have driven much of the recent public and scientific interest in Zika virus (ZIKV), an emerging neuroinvasive flavivirus (Lazear and Diamond 2016). As few effective treatments exist for either adult or congenital neuroinvasive infections, identifying new therapeutic targets for both prevention and intervention is a matter of great interest to investigators (Lazear et al. 2016; Kok 2016). 


\subsection{Programmed Cell Death During Infection}

Among the potential therapeutic targets for neuroinvasive viral infection are the signaling molecules that orchestrate the various forms of programmed cell death. Programmed cell death is a common host response to infection, as it cuts short virus production by infected cells and facilitates key immunological processes such as phagocytosis and antigen presentation (Jorgensen et al. 2017; Brault and Oberst 2017). However, the activation of cell death programs in the CNS involves a unique set of outcomes compared to other tissues. Adult neurons are considered to be predominantly postmitotic and are therefore not easily replaced (Anda et al. 2016). Moreover, individual neurons typically possess highly elaborate and overlapping dendritic arbors whose formation and maintenance rely on both intracellular and intercellular regulation. These synaptic networks are likely permanently compromised following cell death, and, while some redundancy and compensatory mechanisms exist, this loss comes with a significant cost to the organism (Koleske 2013; Klein et al. 2017). Death of myelinating oligodendrocytes is similarly problematic, as structural and biochemical features of the adult CNS prevent the full regeneration of functional myelin sheaths (Franklin and Gallo 2014). Thus, the potential immunological benefits of programmed cell death in the CNS must be weighed against the cost of losing irreplaceable cells and critical cytoarchitecture.

In all tissues, the specific consequences of cell death are influenced by which of the various forms of programmed cell death are triggered (Blander 2014). Apoptosis is the canonical form of programmed cell death, characterized by activation of the caspase proteases and the nonlytic fragmentation and shrinkage of dying cells that are recognized for removal by phagocytes (Jorgensen et al. 2017). While apoptosis can occur in response to infection or stress, it is also the predominant form of developmentally programmed cell death such that the death of cells by apoptosis sculpts developing embryos and contributes to tissue homeostasis throughout life. This elimination of cells that pose no risk to the host proceeds without activating inflammatory effector functions, leading to the impression that apoptosis is immunologically silent (Yamaguchi et al. 2014).

In contrast to apoptosis, programmed cell death pathways leading to cell lysis, such as necroptosis and pyroptosis, occur in response to pathogen infection or cellular stress and do not contribute directly to either development or tissue homeostasis. Both pyroptosis and necroptosis result in the release of intracellular contents as well as the production and release of inflammatory cytokines and other mediators. For these reasons, pyroptosis and necroptosis are considered to be pro-inflammatory. The death of infected cells by these programs likely contributes to initiation of host immune responses (Orozco and Oberst 2017; Bergsbaken et al. 2009). Here, we focus on necroptotic signaling within the CNS.

Necroptosis is defined by activation of receptor-interacting protein kinase (RIPK)3 and RIPK3-dependent phosphorylation of the pseudokinase MLKL (Linkermann and Green 2014). Phosphorylated MLKL can translocate to and apparently disrupt the plasma membrane resulting in cellular swelling and lysis. 
A common form of necroptosis occurs downstream of TNF family death receptors, via RIPK1 recruitment of RIPK3. Two RIPK3-independent pathways may also trigger necroptosis. Toll-like receptor (TLR3) and TLR4 adaptor TRIF and Z-nucleic acid-binding protein (ZBP)1 (also known as DAI and DLM1) may also recruit RIPK3. Unlike either apoptosis or pyroptosis, necroptosis is caspaseindependent and is inhibited by the apoptotic protease caspase- 8 . This feature of necroptotic signaling, combined with the observation that numerous pathogens encode inhibitors of the caspases, has led to the idea that necroptosis represents an alternate form of cell death that is engaged by the inhibition of apoptosis (Mocarski et al. 2014). While this is likely true in some contexts, emerging evidence indicates more complex roles for the necroptotic pathway in immune responses to infection as well as sterile injury (Oberst 2015).

The heterogeneous roles of necroptotic signaling result from differential functions of the RIP kinases, which drive both cell death-dependent and cell-independent signaling outcomes. Notably, the RIP kinases contribute to the initiation of inflammatory transcription pathways, among other functions, in addition to their role in the initiation of necroptosis (Orozco and Oberst 2017). RIPK1 has a well-characterized role in the activation of NF- $\kappa \mathrm{B}$ and MAPK in response to TNF, although this signaling proceeds independently of RIPK1 kinase activity (Silke et al. 2015). Emerging evidence also indicates that RIPK3 contributes to inflammatory responses, independent of the initiation of necroptosis. Supporting this idea is the finding that mice lacking the terminal necroptotic effector MLKL, in which the execution of necroptosis is impossible, differ in phenotype from mice lacking its upstream activator RIPK3, or in which the kinase activity of RIPK1 is ablated (termed Ripk1 ${ }^{K D}$ ) (Newton et al. 2016). Although Ripk3 ${ }^{-/-}$and Ripk1 $1^{K D}$ mice are susceptible to infection by WNV (Daniels et al. 2017b) and influenza (Nogusa et al. 2016), they resist TNF-induced shock and ischemia-reperfusion injury (Newton et al. 2016), phenotypes that are distinct from MLKL-deficient mice. These observations suggest that RIPK3 has additional biological roles beyond activation of MLKL and necroptosis. Thus, the distinction between a role for necroptosis and cell death-independent functions of the RIP kinases within the CNS requires differentiation of outcomes.

Initial work suggests that RIP kinase signaling is a key component of the host response to viruses in the CNS, but that the outcome of this signaling is not necroptosis. In this chapter, we will focus on recent work identifying diverse roles for the necroptotic signaling axis during neuroinvasive viral infection. Beyond the setting of infectious disease, RIPK-driven necroptosis and inflammation have also been implicated in disease associated with various non-infectious neurological pathologies and neurodegenerative disorders. Indeed, a majority of studies of necroptosis within the CNS have been performed using non-infectious disease models (Zhang et al. 2017), with most of these studies identifying necroptosis as highly detrimental to the CNS. Many reports demonstrate remarkable amelioration of CNS inflammation and injury using pharmacological inhibition and/or genetic ablation of RIPK signaling. Thus, we will also discuss recent studies of the impact of necroptosis during non-infectious CNS pathologies, as these studies provide 
important additional insights concerning the immunological outcomes of RIPK signaling and necroptosis within the CNS and potential clues as to how they may function during CNS infection.

\section{Neuroinvasive Viral Infections}

\subsection{Coronaviruses}

Coronaviruses are positive-stranded RNA viruses that are common respiratory pathogens in mammals and birds. However, some coronaviruses, such as human coronavirus OC43 (OC43) and mouse hepatitis virus (MHV), are capable of spreading to the CNS, where they replicate in neurons, resulting in neuronal death and degeneration (Desforges et al. 2014). Coronavirus neuropathology may arise via different mechanisms, including neuroinflammation (Li et al. 2004), unfolded protein responses (Favreau et al. 2009), and glutamate excitotoxicity (Brison et al. 2011). MHV can also chronically persist in the murine CNS, resulting in viral and immune-mediated demyelination (Weiss and Leibowitz 2011). Indeed, coronaviruses are among the infectious agents that have been implicated in idiopathic demyelinating disorders of humans, such as multiple sclerosis (Venkatesan and Johnson 2014; Mentis et al. 2017).

Programmed cell death and RIPK signaling may also drive coronavirus-induced neuropathology. Recent studies suggest that coronaviruses are capable of inducing necroptotic cell death in infected neurons in vitro, and that this neuronal death may contribute to the disease burden observed in mouse models of neuroinvasive coronavirus infection. OC43 infection in primary mouse neurons has been shown to induce RIPK1 and RIPK3 expression, and a mutant strain of OC43 exhibiting increased neurovirulence induced significantly higher expression of both RIPK1 and RIPK3 in neurons compared to a non-mutant reference virus (Meessen-Pinard et al. 2017). Similar results were observed in a differentiated human neuroblastoma cell line (LA-N-5), where OC43 was also shown to induce cell death that was RIPK1- and MLKL-dependent, but Bax- and caspase-independent (Meessen-Pinard et al. 2017; Favreau et al. 2012). While coronavirus-induced neuronal necroptosis in vivo has yet to be reported, the increased propensity for the neurovirulent strain of OC43 to induce neuronal necroptosis in vitro may underlie the greater mortality and neurologic disease burden observed in mouse models using this virus.

One key finding from these studies is that the antiviral outcomes of RIPK signaling in neurons may be separable from the activation of programmed cell death. Knockdown of RIPK1 expression resulted in decreased cell death, along with increased viral replication in OC43-infected LA-N-5 cells (Meessen-Pinard et al. 2017). Remarkably, however, chemical inhibition of MLKL ameliorated cell death but had no significant impact on viral replication, suggesting that cell death-independent outcomes of RIPK1 signaling, such as cytokine production, were 
necessary to restrict viral replication. While the protective versus pathologic outcomes of necroptosis during neuroinvasive coronavirus infection require more extensive study, these initial findings suggest that programmed cell death may not be absolutely necessary for neuronal control of viral replication, and that therapies that target the proximal effectors of cell death while leaving upstream signaling intact may preserve essential immune functions while limiting neuronal injury and degeneration.

\subsection{Japanese Encephalitis Virus}

Flaviviruses are another family of positive-stranded RNA viruses that exhibit neuroinvasive potential. As major encephalitic arboviruses disseminated via insect vectors, most notably mosquitoes, flaviviruses are endemic in most temperate and tropical regions of the world, where seasonal outbreaks and epidemics of viral encephalitis are increasingly common (Salimi et al. 2016). Indeed, flaviviruses are the most common cause of viral encephalitis globally, with Japanese encephalitis virus (JEV) being the most prevalent. JEV infection causes a mild febrile illness in most patients but can result in fatal meningoencephalitis in vulnerable populations. Neuroinvasive JEV infection results in massive neuronal death that is driven by viral replication as well as immunopathology caused by activated glia and infiltrating peripheral leukocytes (Myint et al. 2014; German et al. 2006). Because of this extensive neuronal death, survivors of JEV encephalitis very commonly experience profound and persistent neurologic and psychiatric sequelae (Wang and Liang 2015).

Hallmarks of JEV encephalitis in both rodents and humans are perivascular and parenchymal necrosis within the CNS (German et al. 2006), as well as TNF $\alpha$-dependent neuroinflammation (Chen et al. 2012). Because TNF $\alpha$ is a canonical inducer of necroptosis, these observations suggest that necroptosis may be an important component of the neuroimmune response to JEV. Using a mouse model of JEV encephalitis, Bian and colleagues recently demonstrated considerable upregulation of MLKL expression and phosphorylation within the CNS following JEV infection (Bian et al. 2017). Enhanced MLKL expression was also noted in JEV-infected, $\mathrm{NeuN}^{+}$neurons in vivo, as well as Neuro2a cells in vitro, though direct evidence of MLKL-dependent neuronal cell death was not reported. However, despite robust engagement of MLKL following infection, loss of MLKL did not impact mortality following JEV infection in mice. CNS viral burden was also unchanged in $\mathrm{Mlkl}^{-1-}$ mice compared to WT controls in both intraperitoneal and intracranial infection models. Remarkably, however, although endpoint mortality was unchanged, $M l k l^{-/}$mice exhibited delayed weight loss and progression of clinical signs of disease compared to controls. $M l \mathrm{kl}^{-/-}$mice also exhibited significantly lower CNS expression of inflammatory cytokines, including TNF, IL-1 $\beta$, IFN $\gamma$. These findings suggest that engagement of MLKL within the JEV-infected CNS resulted in neuroinflammation that did not contribute to control of JEV 
replication, but instead worsened disease pathogenesis. Neuroinflammation in response to viral infection commonly results in bystander injury to uninfected neural tissue and, on balance, may be harmful rather than protective (Klein et al. 2017; Swanson and McGavern 2015). Similarly, this initial study suggests that the outcomes of MLKL activation/necroptosis in the JEV-infected CNS also primarily result in immunopathology rather than viral control, and may, in fact, be a promising target for the alleviation of neuroinflammation and neurodegeneration during viral encephalitis. This idea is supported by many recent studies demonstrating the pathological outcomes of necroptosis in the CNS during noninfectious disease states, to be discussed later.

Importantly, although this study examined the impact of the effector protein MLKL during JEV encephalitis, there are other, possibly MLKL-independent, functions of upstream signaling by RIPK1 and RIPK3 in this setting that have not yet been investigated. As mentioned above, RIPK signaling regulates multiple, distinct signaling outcomes, above and beyond the initiation of necroptosis (Meessen-Pinard et al. 2017; Daniels et al. 2017b; Nogusa et al. 2016). RIPKs may, therefore, have additional functions that serve to restrict JEV infection and pathogenesis; these may include induction of apoptosis (Nogusa et al. 2016) as well as cell death-independent contribution to transcription of inflammatory cytokines.

\subsection{West Nile Virus}

Recent work studying RIP kinases in the context of infection with a related flavivirus, West Nile virus (WNV), suggests that inflammatory transcription, rather than necroptotic cell death, may be the prevalent outcome of RIPK activation in the CNS. WNV is included in the JEV serogroup of mosquito-borne flaviviruses of significant global health concern. WNV began to receive significant attention upon its introduction into the USA in 1999 (Lanciotti et al. 1999) and has since become the leading cause of epidemic viral encephalitis, accounting for $>90 \%$ of neuroinvasive arboviral infections each year (Lindsey et al. 2014). Neuroinvasive WNV infection results in considerable viral and immune-mediated pathology, including neuronal cell death (Daniels et al. 2017a; Samuel et al. 2007; Salimi et al. 2016). A high proportion of patients that survive neuroinvasive WNV infection suffer long-lasting cognitive sequelae, with an estimated 10,000 survivors currently living with chronic neurocognitive impairments in the US (Salimi et al. 2016). Chronic impairment appears to result from neuroinflammatory insults that persist beyond the resolution of infection (Vasek et al. 2016; Klein et al. 2017). Despite this high propensity for immunopathology, robust innate and adaptive immune responses are required for effective viral clearance and host survival (Suthar et al. 2013; Cho and Diamond 2012). Thus, as with other neuroinvasive flavivirus infections, distinguishing the mechanisms that promote protective versus pathologic neuroinflammation is a central goal of current research (Klein and Hunter 2017). 
Neuronal apoptosis is a pathologic rather than protective feature of West Nile virus encephalitis, as mice lacking key apoptotic signaling components exhibit decreased tissue injury with no apparent impact on viral dissemination or burden within the CNS (Samuel et al. 2007; Clarke et al. 2014). Recent work in our laboratory sought to investigate necroptotic and non-necroptotic RIPK signaling during WNV infection. In contrast to neuroinvasive coronavirus and JEV, we found no evidence for the initiation of necroptosis following WNV infection (Daniels et al. 2017b). Viral pathogenesis in WNV-infected $M l k l^{-/-}$mice did not significantly differ from WT controls across a broad array of virologic and immunologic parameters. However, both Ripk $3^{-/-}$and Ripk $1^{K D}$ mice exhibited markedly greater susceptibility to WNV, with severe clinical signs of disease and $100 \%$ mortality following subcutaneous infection. This increased susceptibility was ascribed to the absence of RIPK-dependent neuronal chemokine expression and a consequently diminished recruitment of antiviral immune cells to the CNS.

Our study supports the importance of diverse signaling outcomes via RIPK1 and RIPK3, particularly as they affect the immune response in the CNS. Moreover, our study establishes a key protective role for the RIP kinases independent of their canonical role as mediators of programmed cell death. Protection was associated with coordinated chemokine expression but appeared to proceed independently of key inflammatory cytokines, such as TNF and IL1 $\beta$. Chemokines are extraordinarily important neurobiological mediators during both homeostasis and disease, regulating diverse processes that include development, neurotransmission, synaptic maintenance, and myelination, in addition to immunological responses to infection and injury (Williams et al. 2014; Reaux-Le Goazigo et al. 2013; Durrant et al.2014). Our findings suggest that RIPK may serve additional roles in the CNS associated with the coordination of chemokine expression.

\subsection{Zika Virus}

In a subsequent study, we examined roles for the RIP kinases in the control of Zika virus (ZIKV), another mosquito-borne flavivirus that has received significant recent attention due to its global spread, potential for human-to-human transmission, and the severe congenital neurologic pathology associated with vertical transmission from mother to fetus (Miner and Diamond 2017; Pardy and Richer 2019). Unexpectedly, we found that primary cortical neurons derived from mice lacking ZBP1, RIPK3, or the kinase activity of RIPK1 displayed a cell-intrinsic defect in the control of viral replication, while no such defect was observed upon infection of neurons with WNV (Daniels et al. 2019). We traced this cell-intrinsic defect to the metabolic enzyme IRG1, which is upregulated in neurons upon ZIKV infection in a ZBP1- and RIPK-dependent manner. IRG1 catalyzes the production of the metabolite itaconate, which alters cellular metabolism by inhibiting succinate dehydrogenase (SDH) activity (Lampropoulou et al. 2016; Murphy and O'Neill 2018). Suppression of SDH activity by IRG1-dependent itaconate was sufficient to 
reduce ZIKV replication in neurons. Interestingly, we found that the IRG1/itaconate pathway was also able to suppress WNV replication, but that upon WNV infection, IRG1 could be redundantly upregulated by either the ZBP1/RIPK pathway or by the TLR7/Myd88 pathway. These findings implicated alterations in neuronal metabolism as an unexpected adaptation to limit viral replication in these cells, suggesting that these alterations can occur downstream of RIPK activation, among other innate immune signaling pathways.

Though only a handful of studies have examined roles for RIPK signaling and necroptosis in the CNS during viral infection, two key themes have emerged (Fig. 1). First, MLKL-dependent necroptosis is only one possible outcome of RIPK signaling, particularly in neural tissue. On balance, necroptosis appears to be pathologic rather than protective. Second, RIPK signaling in the CNS appears to serve important, protective antiviral and immunologic functions by enhancing inflammatory chemokine expression and altering neuronal metabolism to suppress viral replication and dissemination. An attractive hypothesis to explain these findings is that alternative mechanisms of RIPK-mediated pathogen control have evolved in highly specialized tissues such as the CNS, in which there are significant constraints on repair and in which individual cells serve unique, networked, and irreplaceable functions. Consistent with this idea, in our study of RIPK signaling during WNV encephalitis, we did not observe evidence for death-independent and RIPK-mediated chemokine expression in peripheral myeloid cells. RIPK signaling

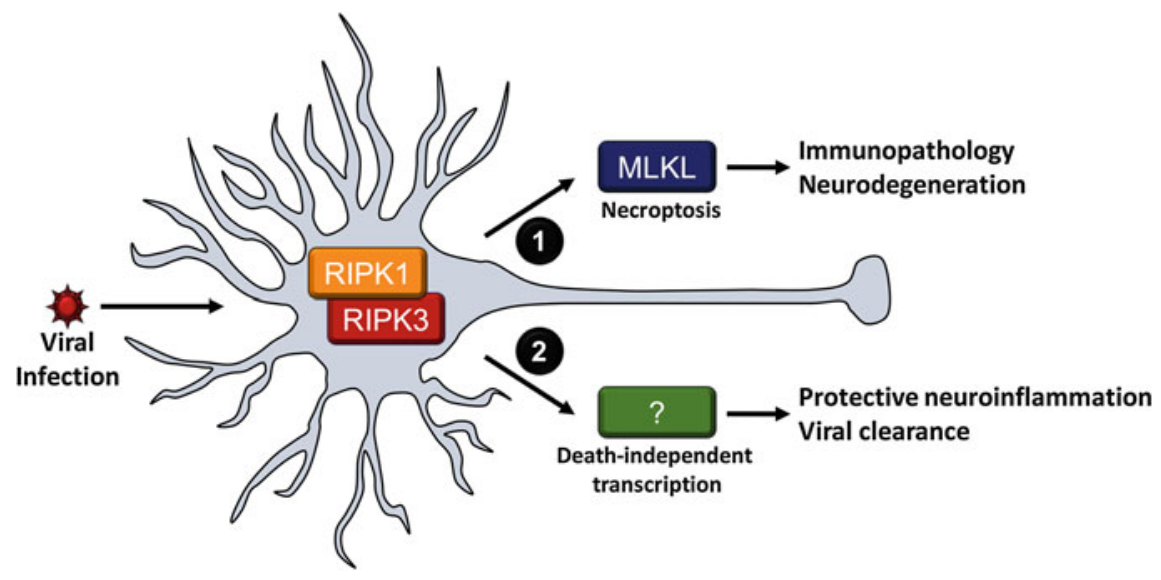

Fig. 1 Diverging outcomes of RIPK signaling during neuroinvasive viral infection. Emerging evidence suggests two possible outcomes of RIPK activation in neurons during viral infection. 1 Some viruses may engage canonical necroptotic cell death via the activation of the executioner protein MLKL. However, MLKL-dependent cell death has so far been shown to be an ineffective antiviral strategy in the CNS, resulting instead in immunopathology and neurodegeneration. 2 RIPK signaling in neurons also appears to serve inflammatory roles that are independent of programmed cell death, though the molecular mechanisms that orchestrate this role remain unknown. Death-independent RIPK signaling has so far been shown to coordinate the transcription of inflammatory chemokines, resulting in protective neuroinflammation and viral clearance 
was dispensable for virologic control and adaptive immunity in peripheral compartments (Daniels et al. 2017b). Consistent with these observations peripheral cells lacking IRG1 did not display the defects in control of ZIKV replication observed in neurons lacking this enzyme (Daniels et al. 2019). Studies in non-CNS model systems have also identified necroptosis-independent outcomes of RIPK signaling, including RIPK-dependent apoptosis and noncanonical inflammasome activation (Nogusa et al. 2016; Najjar et al. 2016; Newton et al. 2016; Lawlor et al. 2015), though neither of these outcomes were observed in the CNS in our studies. Defining general versus CNS-specific outcomes of RIPK signaling during viral infection will be an intriguing area for future study.

\section{Lessons from Noninfectious CNS Pathologies}

Comparatively, more has been done to investigate the roles of RIPK signaling and necroptosis during noninfectious pathologies of the CNS. While there are obvious differences between infection and sterile disease states, CNS injury and neurodegeneration are marked by considerable inflammation and immunopathology, suggesting parallels to infection (Faden et al. 2016; Sankowski et al. 2015). As during CNS infection, necroptosis during noninfectious CNS pathologies also appears to contribute significantly to neurodegeneration and chronic neuroinflammation (Zhang et al. 2017). Recent studies of necroptosis and RIPK signaling in the context of these pathologies provide important additional insight into how these processes function in the CNS more generally and suggest several promising avenues of inquiry for future studies of viral infection.

\subsection{Spinal Cord Injury}

Mechanical trauma to the spinal cord results first in a primary lesion composed of dying cells at the site of trauma, followed by progressive secondary injury to surrounding tissues resulting from ongoing inflammation (Schwab et al. 2014). Cell death within primary lesions has been primarily attributed to non-programmed, mechanically induced necrosis as well as apoptosis (Byrnes et al. 2007). Apoptosis has been observed in both neurons and oligodendrocytes following spinal cord injury (SCI) and can be observed within hours following injury in rodent models (Zhang et al. 2012). The contribution of neuronal and oligodendroglial apoptosis to events that follow SCI is well established, as numerous studies have demonstrated through the use of inhibitors that apoptosis contributes to secondary injury, increased lesion size, and reduced motor function (Han et al. 2012; Yin et al. 2012; Tang et al. 2014; Zhao et al. 2017; Chen et al. 2017a).

Recent evidence, however, suggests that necroptosis also contributes to the pathogenesis of SCI. RIPK3 expression is highly upregulated in both primary and 
secondary SCI lesion tissue and can be observed in neurons, oligodendrocytes, and astrocytes (Kanno et al. 2015; Wu et al. 2016). Moreover, propidium iodide ${ }^{+}$ necrotic cells can be observed within SCI lesions long after the initial mechanical insult, suggesting that ongoing programmed necrosis contributes to secondary injury (Kanno et al. 2015; Fan et al. 2016). Several recent studies have demonstrated that pharmacological blockade of the necroptotic signaling axis significantly improves outcomes following SCI. For example, administration of the canonical RIPK1 inhibitor necrostatin-1 (Nec-1) has been shown to effectively increase neuronal survival, reduce tissue damage, and improve recovery of motor function in rodent SCI models (Liu et al. 2015; Wang et al. 2014). Pharmacological inhibition of necroptosis may also prevent injury to the structural tissues of the spine, such as the nucleus pulposus of the intravertebral disk (Chen et al. 2017b).

In addition to serving as a proximal mechanism of neuronal pathogenesis following primary injury, necroptosis also appears to contribute to secondary injury via multicellular processes. In particular, necroptosis within inflammatory M1 microglia and reactive astrocytes has been associated with ongoing immunopathology and poor outcomes following SCI (Fan et al. 2016). A hallmark of secondary injury in the CNS is the initiation of reactive gliosis, characterized in part by the proliferation of microglia, astrocytes, and oligodendrocyte precursor cells (OPCs) within and around lesion tissue. Gliosis is a complex process that has both beneficial and detrimental effects at the site of injury. Proliferating glia promote a return to homeostasis within injured tissue by inhibiting excitotoxicity, restoring blood-brain barrier function, and physically sequestering injured tissue from adjacent healthy tissue via formation of a "glial scar." However, gliosis and glial scar formation also actively inhibit axon regeneration and contribute to the formation of "cavities" around injured tissue that preclude functional recovery (Burda and Sofroniew 2014).

Recent studies demonstrate that reactive glia undergo multiple forms of programmed cell death, including necroptosis, during gliosis and that different cell death programs contribute to the diverse regulatory outcomes of gliosis following SCI. For example, inflammatory M1 macrophages and microglia have been shown to induce necroptosis in reactive astrocytes, and astrocytic necroptosis, in turn, contributes to immunopathology and spinal cord cavitation (Fan et al. 2016). Inhibition of necroptosis in a rodent model of SCI has been shown to not only significantly ameliorate injury and cavitation, but also to skew the gene expression of reactive astrocytes toward a beneficial, neurotrophic phenotype (Fan et al. 2016). Necroptotic M1 microglia have also been observed in spinal cord lesions in both rodents and humans, where they likely also contribute to ongoing neuroinflammation and injury (Fan et al. 2015, 2016; Dhib-Jalbut and Kalvakolanu 2015). These studies and others establish that necroptotic glia contribute significantly to ongoing inflammation and neuropathology during CNS injury and may serve as important additional targets for clinical intervention during neuroinfectious diseases in which reactive gliosis contributes to neuropathogenesis (Burda and Sofroniew 2014). 


\subsection{Traumatic Brain Injury}

Similarly to SCI, trauma to the higher CNS also results in a biphasic response of primary cell death followed by neuroinflammation and secondary injury. In contrast, to SCI, however, traumatic brain injury (TBI) has fewer options for surgical intervention, and, in addition to the sensorimotor impairments observed following SCI, TBI can result in long-term cognitive, emotional, and behavioral dysfunction (Walker and Tesco 2013). Moreover, TBI is a purported risk factor for the later development of neurodegenerative diseases, including Alzheimer's disease, Parkinson's disease, and Amyotrophic Lateral Sclerosis (ALS) (Gardner and Yaffe 2015; Faden and Loane 2015). Emerging evidence demonstrates that several classes of neural cells are susceptible to necroptosis following TBI (Walker and Tesco 2013). RIPK1, RIPK3, and MLKL expression have been shown to increase in forebrain regions including cerebral cortex and hippocampus in rodent controlled cortical impact (CCI) models (Zhou et al. 2012; Liu et al. 2016), and Nec-1 administration has been shown to significantly reduce neuroinflammation and ameliorate functional impairment following CCI (You et al. 2008; Wang et al. 2012).

Notably, studies of RIPK signaling during TBI suggest that necroptosis can occur in adult neural progenitor cells. Using a CCI model, Zhou and colleagues observed that the majority of degenerating cells in the hippocampal dentate gyrus, a major site of adult neurogenesis, were immature granular neurons (Zhou et al. 2012). Most of these dying immature neurons did not exhibit classical morphological and biochemical characteristics of apoptosis, but did exhibit high levels of RIPK1 expression. While more explicit confirmation of RIPK-mediated necroptosis in neuronal progenitors is needed, this study, combined with others demonstrating improved functional recovery from TBI following pharmacological blockade of RIPK1 (You et al. 2008; Wang et al. 2012), suggests that RIPK signaling may further exacerbate neurologic disease by inhibiting CNS repair. Perturbations of neurogenesis during neuroinvasive infections of both fetuses and adults have been described in recent reports, including several notable studies of ZIKV infection, and likely contribute to long-term cognitive and functional impairments observed in survivors of these infections (Sun et al. 2014; Liang et al. 2016; Bayless et al. 2016; Brnic et al. 2012; Li et al. 2016; Tang et al. 2016). Thus, studies examining the susceptibility of neural progenitors to necroptosis during viral infection are warranted and may yield important new insight into the poorly understood etiology of microcephaly and other neurologic impairments resulting from ZIKV infection.

\subsection{Neurodegeneration}

The CNS is also susceptible to chronic neurodegenerative diseases such as Alzheimer's Disease and Parkinson's Disease, which remain poorly understood despite widespread public awareness. Neurodegenerative disease is most often 
observed in aging populations and is characterized by the progressive atrophy and death of neurons, ultimately manifesting in motor dysfunction, dementia, or other significant neurologic impairments (Hindle 2010; Reitz and Mayeux 2014). Several distinct stimuli have been found to trigger neurodegeneration, including dysregulated protein folding, axonal transport, and lysosomal storage, among others (Bourdenx et al. 2017; Onyenwoke and Brenman 2015). The pathophysiological mechanisms of neurodegeneration are also complex and include excitotoxicity (Mehta et al. 2013), inflammation (Sankowski et al. 2015), and programmed cell death (Fan et al. 2017). Remarkably, despite the extraordinary heterogeneity of these diseases, a growing body of recent research has established that pharmacological inhibition of RIPK1 signaling ameliorates pathology in a diverse set of neurodegenerative disease models, including models of Alzheimer's Disease (Yang et al. 2017; Qinli et al. 2013), Parkinson's Disease (Wu et al. 2015; Dionisio et al. 2019), ALS (Ito et al. 2016; Re et al. 2014), Huntington's Disease (Zhu et al. 2011), and others (Cougnoux et al. 2016; Zhang et al. 2017), though some of these findings remain controversial (Wang et al. 2019). While not all of these studies directly implicate necroptosis as a pathological driver of neurodegeneration, it is now clear that RIPK signaling is capable of orchestrating several distinct degenerative processes in the CNS, through programmed cell death, neuroinflammation, and other processes (Zhang et al. 2017).

One particularly intriguing finding of recent studies of necroptosis during neurodegeneration is the apparent central involvement of RIPK signaling across a number of neuronal subtypes and brain regions. The brain exhibits remarkable immunologic heterogeneity, as cell type and region-specific immune responses have been observed during both neuroinvasive infection and CNS autoimmunity (Daniels et al. 2017a; Cho et al. 2013; Stromnes et al. 2008; Lees et al. 2008; Durrant et al. 2016; Williams et al. 2020). However, inhibition or genetic ablation of RIPK signaling has been shown to limit degeneration and/or cell death in diverse neuronal populations, including cultures of spinal cord motor neurons ( $R e$ et al. 2014; Ito et al. 2016), cerebral cortical neurons (Qinli et al. 2013), striatal neurons (Zhu et al. 2011), and dopaminergic PC12 cells (Wu et al. 2015). Similar findings have been observed in vivo across several functionally and anatomically distinct CNS regions, including the cerebral cortex (Yang et al. 2017; Vitner et al. 2014), hippocampus (Yang et al. 2017), cerebellum (Cougnoux et al. 2016), and spinal cord (Ito et al. 2016). These findings implicate RIPK signaling as a central driver of pathological processes across a broad set of CNS tissues with diverse functional profiles and developmental ontogenies.

The implication of this signaling axis in the pathogenesis of neurodegenerative disease across the CNS suggests that RIPK signaling may also be a highly relevant mediator of host responses to a broad array of neuroinvasive infections targeting many different CNS regions and cell types. However, the central involvement of this pathway across CNS pathologies may complicate efforts to target it for therapeutic intervention. On one hand, the necroptotic signaling axis may provide broad-spectrum targets that improve the feasibility of therapeutic development for neuroinvasive viral infections. Neuroinvasive viruses collectively represent a major 
source of human disease burden; however, individual viral species often cause only a small number of clinical cases, limiting the cost-effectiveness of developing virus-specific therapies (Kok 2016). Therapeutic intervention with RIPK inhibitors to treat neurodegeneration may also increase susceptibility to neuroinvasive viral infection. Further work uncovering how these processes operate across different CNS cell types, regions, and pathogens will be necessary to guide the development and delivery of effective therapies.

\section{Conclusions}

Necroptosis has been traditionally understood as an alternative, immunogenic form of programmed cell death and an important component of the host response to pathogens. Emerging evidence suggests, however, that necroptosis during acute viral infection of the CNS is, at best, an ineffective antiviral response and, at worst, a significant driver of neuropathology. This idea is supported by a larger body of evidence demonstrating the diverse contributions of RIPK signaling and necroptosis to the neuropathogenesis of CNS injury and neurodegeneration. Despite this detrimental role, the RIP kinases also appear to coordinate unexpected, necroptosis-independent antiviral immune responses in the CNS, promoting protective rather than pathological neuroinflammation.

Future research should work to more fully uncover the extent to which RIPK signaling serves specialized functions in the CNS during viral infection, as well as the physiologic and evolutionary drivers of these functions. The continuing development of improved genetic and other tools, such as Ripk $3^{\text {flox/flox }}$ mice (Strilic et al. 2016), will allow more careful examination of cell- and tissue-specific roles for the necroptotic signaling axis across experimental systems. Studies using a broader range of neurotropic viruses are also needed, as this pathway may function differently in response to variations in pathogen detection, route of neuroinvasion, or differential cellular tropism of individual viruses. Understanding the specific mechanisms that govern RIPK-driven neuroimmune responses may be of great benefit in the study of neuroinvasive viral infection, for which new molecular targets for prevention and therapy are urgently needed.

\section{References}

Anda FC, Madabhushi R, Rei D, Meng J, Graff J, Durak O, Meletis K, Richter M, Schwanke B, Mungenast A, Tsai LH (2016) Cortical neurons gradually attain a post-mitotic state. Cell Res 26(9):1033-1047

Bayless NL, Greenberg RS, Swigut T, Wysocka J, Blish CA (2016) Zika virus infection induces cranial neural crest cells to produce cytokines at levels detrimental for neurogenesis. Cell Host Microbe 20(4):423-428 
Bergsbaken T, Fink SL, Cookson BT (2009) Pyroptosis: host cell death and inflammation. Nat Rev Microbiol 7(2):99-109

Bian P, Zheng X, Wei L, Ye C, Fan H, Cai Y, Zhang Y, Zhang F, Jia Z, Lei Y (2017) MLKL Mediated necroptosis accelerates JEV-induced neuroinflammation in mice. Frontiers in microbiology 8:303

Blander JM (2014) A long-awaited merger of the pathways mediating host defence and programmed cell death. Nat Rev Immunol 14(9):601-618

Bourdenx M, Koulakiotis NS, Sanoudou D, Bezard E, Dehay B, Tsarbopoulos A (2017) Protein aggregation and neurodegeneration in prototypical neurodegenerative diseases: examples of amyloidopathies, tauopathies and synucleinopathies. Prog Neurobiol 155:171-193

Brault M, Oberst A (2017) Controlled detonation: evolution of necroptosis in pathogen defense. Immunol Cell Biol 95(2):131-136

Brison E, Jacomy H, Desforges M, Talbot PJ (2011) Glutamate excitotoxicity is involved in the induction of paralysis in mice after infection by a human coronavirus with a single point mutation in its spike protein. J Virol 85(23):12464-12473

Brnic D, Stevanovic V, Cochet M, Agier C, Richardson J, Montero-Menei CN, Milhavet O, Eloit M, Coulpier M (2012) Borna disease virus infects human neural progenitor cells and impairs neurogenesis. J Virol 86(5):2512-2522

Burda JE, Sofroniew MV (2014) Reactive gliosis and the multicellular response to CNS damage and disease. Neuron 81(2):229-248

Byrnes KR, Stoica BA, Fricke S, Di Giovanni S, Faden AI (2007) Cell cycle activation contributes to post-mitotic cell death and secondary damage after spinal cord injury. Brain $130(\mathrm{Pt}$ 11):2977-2992

Chen CJ, Ou YC, Chang CY, Pan HC, Liao SL, Chen SY, Raung SL, Lai CY (2012) Glutamate released by Japanese encephalitis virus-infected microglia involves TNF-alpha signaling and contributes to neuronal death. Glia 60(3):487-501

Chen H, Ji H, Zhang M, Liu Z, Lao L, Deng C, Chen J, Zhong G (2017a) An agonist of the protective factor SIRT1 Improves functional recovery and promotes neuronal survival by attenuating inflammation after spinal cord injury. J Neurosci 37(11):2916-2930

Chen S, Lv X, Hu B, Shao Z, Wang B, Ma K, Lin H, Cui M (2017b) RIPK1/RIPK3/ MLKL-mediated necroptosis contributes to compression-induced rat nucleus pulposus cells death. Apoptosis 22(5):626-638

Cho H, Diamond MS (2012) Immune responses to West Nile virus infection in the central nervous system. Viruses 4(12):3812-3830

Cho H, Proll SC, Szretter KJ, Katze MG, Gale M Jr, Diamond MS (2013) Differential innate immune response programs in neuronal subtypes determine susceptibility to infection in the brain by positive-stranded RNA viruses. Nat Med 19(4):458-464

Clarke P, Leser JS, Quick ED, Dionne KR, Beckham JD, Tyler KL (2014) Death receptor-mediated apoptotic signaling is activated in the brain following infection with West Nile virus in the absence of a peripheral immune response. J Virol 88(2):1080-1089

Cougnoux A, Cluzeau C, Mitra S, Li R, Williams I, Burkert K, Xu X, Wassif CA, Zheng W, Porter FD (2016) Necroptosis in Niemann-Pick disease, type C1: a potential therapeutic target. Cell Death Dis 7:e2147

Coyne CB, Lazear HM (2016) Zika virus-reigniting the TORCH. Nat Rev Microbiol 14 (11):707-715

Daniels BP, Jujjavarapu H, Durrant DM, Williams JL, Green RR, White JP, Lazear HM, Gale M Jr, Diamond MS, Klein RS (2017a) Regional astrocyte IFN signaling restricts pathogenesis during neurotropic viral infection. J Clin Invest 127(3):843-856

Daniels BP, Klein RS (2015) Viral sensing at the blood-brain barrier: new roles for innate immunity at the CNS vasculature. Clin Pharmacol Ther 97(4):372-379

Daniels BP, Kofman SB, Smith JR, Norris GT, Snyder AG, Kolb JP, Gao X, Locasale JW, Martinez J, Gale M, Jr, Loo YM, Oberst A (2019) The Nucleotide sensor ZBP1 and kinase RIPK3 Induce the enzyme IRG1 to promote an antiviral metabolic state in neurons. Immunity 50(1):64-76, e64 
Daniels BP, Snyder AG, Olsen TM, Orozco S, Oguin TH, 3rd, Tait SW, Martinez J, Gale M, Jr, Loo YM, Oberst A (2017b) RIPK3 restricts viral pathogenesis via cell death-independent neuroinflammation. Cell 169(2):301-313 e311

Desforges M, Le Coupanec A, Brison E, Meessen-Pinard M, Talbot PJ (2014) Neuroinvasive and neurotropic human respiratory coronaviruses: potential neurovirulent agents in humans. Adv Exp Med Biol 807:75-96

Dhib-Jalbut S, Kalvakolanu DV (2015) Microglia and necroptosis: the culprits of neuronal cell death in multiple sclerosis. Cytokine 76(2):583-584

Dionisio PA, Oliveira SR, Gaspar MM, Gama MJ, Castro-Caldas M, Amaral JD, Rodrigues CMP (2019) Ablation of RIP3 protects from dopaminergic neurodegeneration in experimental Parkinson's disease. Cell Death Dis 10(11):840

Durrant DM, Ghosh S, Klein RS (2016) The olfactory bulb: an immunosensory effector organ during neurotropic viral infections. ACS Chem Neurosci 7(4):464-469

Durrant DM, Williams JL, Daniels BP, Klein RS (2014) Chemokines referee inflammation within the central nervous system during infection and disease. Adv Med 2014:806741

Faden AI, Loane DJ (2015) Chronic neurodegeneration after traumatic brain injury: Alzheimer disease, chronic traumatic encephalopathy, or persistent neuroinflammation? Neurotherapeutics 12(1):143-150

Faden AI, Wu J, Stoica BA, Loane DJ (2016) Progressive inflammation-mediated neurodegeneration after traumatic brain or spinal cord injury. Br J Pharmacol 173(4):681-691

Fan H, Tang HB, Kang J, Shan L, Song H, Zhu K, Wang J, Ju G, Wang YZ (2015) Involvement of endoplasmic reticulum stress in the necroptosis of microglia/macrophages after spinal cord injury. Neuroscience 311:362-373

Fan H, Zhang K, Shan L, Kuang F, Chen K, Zhu K, Ma H, Ju G, Wang YZ (2016) Reactive astrocytes undergo M1 microglia/macrohpages-induced necroptosis in spinal cord injury. Mol Neurodegener 11:14

Fan J, Dawson TM, Dawson VL (2017) Cell death mechanisms of neurodegeneration. Adv Neurobiol 15:403-425

Favreau DJ, Desforges M, St-Jean JR, Talbot PJ (2009) A human coronavirus OC43 variant harboring persistence-associated mutations in the $\mathrm{S}$ glycoprotein differentially induces the unfolded protein response in human neurons as compared to wild-type virus. Virology 395 (2):255-267

Favreau DJ, Meessen-Pinard M, Desforges M, Talbot PJ (2012) Human coronavirus-induced neuronal programmed cell death is cyclophilin $\mathrm{d}$ dependent and potentially caspase dispensable. J Virol 86(1):81-93

Franklin RJ, Gallo V (2014) The translational biology of remyelination: past, present, and future. Glia 62(11):1905-1915

Gardner RC, Yaffe K (2015) Epidemiology of mild traumatic brain injury and neurodegenerative disease. Mol Cell Neurosci 66(Pt B):75-80

German AC, Myint KS, Mai NT, Pomeroy I, Phu NH, Tzartos J, Winter P, Collett J, Farrar J, Barrett A, Kipar A, Esiri MM, Solomon T (2006) A preliminary neuropathological study of Japanese encephalitis in humans and a mouse model. Trans R Soc Trop Med Hyg 100 (12):1135-1145

Han X, Lu M, Wang S, Lv D, Liu H (2012) Targeting IKK/NF-kappaB pathway reduces infiltration of inflammatory cells and apoptosis after spinal cord injury in rats. Neurosci Lett 511(1):28-32

Hindle JV (2010) Ageing, neurodegeneration and Parkinson's disease. Age Ageing 39(2):156-161

Ito Y, Ofengeim D, Najafov A, Das S, Saberi S, Li Y, Hitomi J, Zhu H, Chen H, Mayo L, Geng J, Amin P, DeWitt JP, Mookhtiar AK, Florez M, Ouchida AT, Fan JB, Pasparakis M, Kelliher MA, Ravits J, Yuan J (2016) RIPK1 mediates axonal degeneration by promoting inflammation and necroptosis in ALS. Science 353(6299):603-608

Jorgensen I, Rayamajhi M, Miao EA (2017) Programmed cell death as a defence against infection. Nat Rev Immunol 17(3):151-164 
Kallianpur AR, Levine AJ (2014) Host genetic factors predisposing to HIV-associated neurocognitive disorder. Curr HIV/AIDS Rep 11(3):336-352

Kanno H, Ozawa H, Tateda S, Yahata K, Itoi E (2015) Upregulation of the receptor-interacting protein 3 expression and involvement in neural tissue damage after spinal cord injury in mice. BMC Neurosci 16:62

Klein RS, Garber C, Howard N (2017) Infectious immunity in the central nervous system and brain function. Nat Immunol 18(2):132-141

Klein RS, Hunter CA (2017) Protective and pathological immunity during central nervous system infections. Immunity 46(6):891-909

Kok WM (2016) New developments in flavivirus drug discovery. Expert Opin Drug Discov 1-13

Koleske AJ (2013) Molecular mechanisms of dendrite stability. Nat Rev Neurosci 14(8):536-550

Lampropoulou V, Sergushichev A, Bambouskova M, Nair S, Vincent EE, Loginicheva E, Cervantes-Barragan L, Ma X, Huang SC, Griss T, Weinheimer CJ, Khader S, Randolph GJ, Pearce EJ, Jones RG, Diwan A, Diamond MS, Artyomov MN (2016) Itaconate links inhibition of succinate dehydrogenase with macrophage metabolic remodeling and regulation of inflammation. Cell Metab 24(1):158-166

Lanciotti RS, Roehrig JT, Deubel V, Smith J, Parker M, Steele K, Crise B, Volpe KE, Crabtree MB, Scherret JH, Hall RA, MacKenzie JS, Cropp CB, Panigrahy B, Ostlund E, Schmitt B, Malkinson M, Banet C, Weissman J, Komar N, Savage HM, Stone W, McNamara T, Gubler DJ (1999) Origin of the West Nile virus responsible for an outbreak of encephalitis in the Northeastern United States. Science 286(5448):2333-2337

Lawlor KE, Khan N, Mildenhall A, Gerlic M, Croker BA, D'Cruz AA, Hall C, Kaur Spall S, Anderton H, Masters SL, Rashidi M, Wicks IP, Alexander WS, Mitsuuchi Y, Benetatos CA, Condon SM, Wong WW, Silke J, Vaux DL, Vince JE (2015) RIPK3 promotes cell death and NLRP3 inflammasome activation in the absence of MLKL. Nat Commun 6:6282

Lazear HM, Diamond MS (2016) Zika virus: new clinical syndromes and its emergence in the Western Hemisphere. J Virol 90(10):4864-4875

Lazear HM, Stringer EM, de Silva AM (2016) The emerging Zika virus epidemic in the americas: research priorities. JAMA 315(18):1945-1946

Lees JR, Golumbek PT, Sim J, Dorsey D, Russell JH (2008) Regional CNS responses to IFN-gamma determine lesion localization patterns during EAE pathogenesis. J Exp Med 205 (11):2633-2642

Li H, Saucedo-Cuevas L, Regla-Nava JA, Chai G, Sheets N, Tang W, Terskikh AV, Shresta S, Gleeson JG (2016) Zika virus infects neural progenitors in the adult mouse brain and alters proliferation. Cell Stem Cell 19(5):593-598

Li Y, Fu L, Gonzales DM, Lavi E (2004) Coronavirus neurovirulence correlates with the ability of the virus to induce proinflammatory cytokine signals from astrocytes and microglia. J Virol 78 (7):3398-3406

Liang Q, Luo Z, Zeng J, Chen W, Foo SS, Lee SA, Ge J, Wang S, Goldman SA, Zlokovic BV, Zhao Z, Jung JU (2016) Zika virus NS4A and NS4B proteins deregulate Akt-mTOR signaling in human fetal neural stem cells to inhibit neurogenesis and induce autophagy. Cell Stem Cell 19(5):663-671

Lindsey NP, Lehman JA, Staples JE, Fischer M (2015) West Nile Virus and other nationally notifiable arboviral diseases-United States, 2014. MMWR Morb Mortal Wkly Rep 64 (34):929-934

Linkermann A, Green DR (2014) Necroptosis. N Engl J Med 370(5):455-465

Liu M, Wu W, Li H, Li S, Huang LT, Yang YQ, Sun Q, Wang CX, Yu Z, Hang CH (2015) Necroptosis, a novel type of programmed cell death, contributes to early neural cells damage after spinal cord injury in adult mice. J Spinal Cord Med 38(6):745-753

Liu T, Zhao DX, Cui H, Chen L, Bao YH, Wang Y, Jiang JY (2016) Therapeutic hypothermia attenuates tissue damage and cytokine expression after traumatic brain injury by inhibiting necroptosis in the rat. Sci Rep 6:24547 
Long D, Deng X, Singh P, Loeb M, Lauring AS, Seielstad M (2016) Identification of genetic variants associated with susceptibility to West Nile virus neuroinvasive disease. Genes Immun 17(5):298-304

Meessen-Pinard M, Le Coupanec A, Desforges M, Talbot PJ (2017) Pivotal Role of Receptor-Interacting Protein Kinase 1 and Mixed Lineage Kinase Domain-Like in Neuronal Cell Death Induced by the Human Neuroinvasive Coronavirus OC43. J Virol 91(1)

Mehta A, Prabhakar M, Kumar P, Deshmukh R, Sharma PL (2013) Excitotoxicity: bridge to various triggers in neurodegenerative disorders. Eur J Pharmacol 698(1-3):6-18

Mentis AA, Dardiotis E, Grigoriadis N, Petinaki E, Hadjigeorgiou GM (2017) Viruses and endogenous retroviruses in multiple sclerosis: from correlation to causation. Acta Neurol Scand

Miner JJ, Diamond MS (2017) Zika virus pathogenesis and tissue tropism. Cell Host Microbe 21 (2):134-142

Mocarski ES, Kaiser WJ, Livingston-Rosanoff D, Upton JW, Daley-Bauer LP (2014) True grit: programmed necrosis in antiviral host defense, inflammation, and immunogenicity. J Immunol 192(5):2019-2026

Murphy MP, O'Neill LAJ (2018) Krebs cycle reimagined: the emerging roles of succinate and itaconate as signal transducers. Cell 174(4):780-784

Murray K, Baraniuk S, Resnick M, Arafat R, Kilborn C, Cain K, Shallenberger R, York TL, Martinez D, Hellums JS, Hellums D, Malkoff M, Elgawley N, McNeely W, Khuwaja SA, Tesh RB (2006) Risk factors for encephalitis and death from West Nile virus infection. Epidemiol Infect 134(6):1325-1332

Myint KS, Kipar A, Jarman RG, Gibbons RV, Perng GC, Flanagan B, Mongkolsirichaikul D, Van Gessel Y, Solomon T (2014) Neuropathogenesis of Japanese encephalitis in a primate model. PLoS Negl Trop Dis 8(8):e2980

Najjar M, Saleh D, Zelic M, Nogusa S, Shah S, Tai A, Finger JN, Polykratis A, Gough PJ, Bertin J, Whalen MJ, Pasparakis M, Balachandran S, Kelliher M, Poltorak A, Degterev A (2016) RIPK1 and RIPK3 kinases promote cell-death-independent inflammation by toll-like receptor 4. Immunity

Neu N, Duchon J, Zachariah P (2015) TORCH infections. Clin Perinatol 42(1):77-103, viii

Newton K, Dugger DL, Maltzman A, Greve JM, Hedehus M, Martin-McNulty B, Carano RA, Cao TC, van Bruggen N, Bernstein L, Lee WP, Wu X, DeVoss J, Zhang J, Jeet S, Peng I, McKenzie BS, Roose-Girma M, Caplazi P, Diehl L, Webster JD, Vucic D (2016) RIPK3 deficiency or catalytically inactive RIPK1 provides greater benefit than MLKL deficiency in mouse models of inflammation and tissue injury. Cell Death Differentiation

Nogusa S, Thapa RJ, Dillon CP, Liedmann S, Oguin TH 3rd, Ingram JP, Rodriguez DA, Kosoff R, Sharma S, Sturm O, Verbist K, Gough PJ, Bertin J, Hartmann BM, Sealfon SC, Kaiser WJ, Mocarski ES, Lopez CB, Thomas PG, Oberst A, Green DR, Balachandran S (2016) RIPK3 activates parallel pathways of MLKL-driven necroptosis and FADD-mediated apoptosis to protect against influenza a virus. Cell Host Microbe 20(1):13-24

Oberst A (2015) Death in the fast lane: what's next for necroptosis? FEBS J

Onyenwoke RU, Brenman JE (2015) Lysosomal storage diseases-regulating neurodegeneration. J Exp Neurosci 9(Suppl 2):81-91

Orozco S, Oberst A (2017) RIPK3 in cell death and inflammation: the good, the bad, and the ugly. Immunol Rev 277(1):102-112

Pardy RD, Richer MJ (2019) Zika virus pathogenesis: from early case reports to epidemics. Viruses 11(10)

Patel H, Sander B, Nelder MP (2015) Long-term sequelae of West Nile virus-related illness: a systematic review. Lancet Infect Dis 15(8):951-959

Qinli Z, Meiqing L, Xia J, Li X, Weili G, Xiuliang J, Junwei J, Hailan Y, Ce Z, Qiao N (2013) Necrostatin-1 inhibits the degeneration of neural cells induced by aluminum exposure. Restor Neurol Neurosci 31(5):543-555

Re DB, Le Verche V, Yu C, Amoroso MW, Politi KA, Phani S, Ikiz B, Hoffmann L, Koolen M, Nagata T, Papadimitriou D, Nagy P, Mitsumoto H, Kariya S, Wichterle H, Henderson CE, 
Przedborski S (2014) Necroptosis drives motor neuron death in models of both sporadic and familial ALS. Neuron 81(5):1001-1008

Reaux-Le Goazigo A, Van Steenwinckel J, Rostene W, Melik Parsadaniantz S (2013) Current status of chemokines in the adult CNS. Prog Neurobiol 104:67-92

Reitz C, Mayeux R (2014) Alzheimer disease: epidemiology, diagnostic criteria, risk factors and biomarkers. Biochem Pharmacol 88(4):640-651

Ronca SE, Dineley KT, Paessler S (2016) Neurological sequelae resulting from encephalitic alphavirus infection. Frontiers in microbiology 7:959

Salimi H, Cain MD, Klein RS (2016) Encephalitic arboviruses: emergence, clinical presentation, and neuropathogenesis. Neurotherapeutics 13(3):514-534

Samuel MA, Morrey JD, Diamond MS (2007) Caspase 3-dependent cell death of neurons contributes to the pathogenesis of West Nile virus encephalitis. J Virol 81(6):2614-2623

Sankowski R, Mader S, Valdes-Ferrer SI (2015) Systemic inflammation and the brain: novel roles of genetic, molecular, and environmental cues as drivers of neurodegeneration. Front Cell Neurosci 9:28

Schwab JM, Zhang Y, Kopp MA, Brommer B, Popovich PG (2014) The paradox of chronic neuroinflammation, systemic immune suppression, autoimmunity after traumatic chronic spinal cord injury. Exp Neurol 258:121-129

Silke J, Rickard JA, Gerlic M (2015) The diverse role of RIP kinases in necroptosis and inflammation. Nat Immunol 16(7):689-697

Singh TD, Fugate JE, Rabinstein AA (2015) The spectrum of acute encephalitis: causes, management, and predictors of outcome. Neurology 84(4):359-366

Strilic B, Yang L, Albarran-Juarez J, Wachsmuth L, Han K, Muller UC, Pasparakis M, Offermanns S (2016) Tumour-cell-induced endothelial cell necroptosis via death receptor 6 promotes metastasis. Nature 536(7615):215-218

Stromnes IM, Cerretti LM, Liggitt D, Harris RA, Goverman JM (2008) Differential regulation of central nervous system autoimmunity by $\mathrm{T}(\mathrm{H}) 1$ and $\mathrm{T}(\mathrm{H}) 17$ cells. Nat Med 14(3):337-342

Sun T, Vasek MJ, Klein RS (2014) Congenitally acquired persistent lymphocytic choriomeningitis viral infection reduces neuronal progenitor pools in the adult hippocampus and subventricular zone. PLoS ONE 9(5):e96442

Suthar MS, Diamond MS, Gale M Jr (2013) West Nile virus infection and immunity. Nat Rev Microbiol 11(2):115-128

Swanson PA 2nd, McGavern DB (2015) Viral diseases of the central nervous system. Curr Opin Virol 11:44-54

Tang H, Hammack C, Ogden SC, Wen Z, Qian X, Li Y, Yao B, Shin J, Zhang F, Lee EM, Christian KM, Didier RA, Jin P, Song H, Ming GL (2016) Zika virus infects human cortical neural progenitors and attenuates their growth. Cell Stem Cell 18(5):587-590

Tang P, Hou H, Zhang L, Lan X, Mao Z, Liu D, He C, Du H, Zhang L (2014) Autophagy reduces neuronal damage and promotes locomotor recovery via inhibition of apoptosis after spinal cord injury in rats. Mol Neurobiol 49(1):276-287

Vasek MJ, Garber C, Dorsey D, Durrant DM, Bollman B, Soung A, Yu J, Perez-Torres C, Frouin A, Wilton DK, Funk K, DeMasters BK, Jiang X, Bowen JR, Mennerick S, Robinson JK, Garbow JR, Tyler KL, Suthar MS, Schmidt RE, Stevens B, Klein RS (2016) A complement-microglial axis drives synapse loss during virus-induced memory impairment. Nature 534(7608):538-543

Venkatesan A, Johnson RT (2014) Infections and multiple sclerosis. Handb Clin Neurol 122:151-171

Vitner EB, Salomon R, Farfel-Becker T, Meshcheriakova A, Ali M, Klein AD, Platt FM, Cox TM, Futerman AH (2014) RIPK3 as a potential therapeutic target for Gaucher's disease. Nat Med 20(2):204-208

Walker KR, Tesco G (2013) Molecular mechanisms of cognitive dysfunction following traumatic brain injury. Front Aging Neurosci 5:29

Wang H, Liang G (2015) Epidemiology of Japanese encephalitis: past, present, and future prospects. Ther Clin Risk Manag 11:435-448 
Wang T, Perera ND, Chiam MDF, Cuic B, Wanniarachchillage N, Tomas D, Samson AL, Cawthorne W, Valor EN, Murphy JM, Turner BJ (2019) Necroptosis is dispensable for motor neuron degeneration in a mouse model of ALS. Cell Death Differ

Wang Y, Wang H, Tao Y, Zhang S, Wang J, Feng X (2014) Necroptosis inhibitor necrostatin-1 promotes cell protection and physiological function in traumatic spinal cord injury. Neuroscience 266:91-101

Wang YQ, Wang L, Zhang MY, Wang T, Bao HJ, Liu WL, Dai DK, Zhang L, Chang P, Dong WW, Chen XP, Tao LY (2012) Necrostatin-1 suppresses autophagy and apoptosis in mice traumatic brain injury model. Neurochem Res 37(9):1849-1858

Weiss SR, Leibowitz JL (2011) Coronavirus pathogenesis. Adv Virus Res 81:85-164

Williams JL, Holman DW, Klein RS (2014) Chemokines in the balance: maintenance of homeostasis and protection at CNS barriers. Front Cell Neurosci 8:154

Williams JL, Manivasagam S, Smith BC, Sim J, Vollmer LL, Daniels BP, Russell JH, Klein RS (2020) Astrocyte-T cell crosstalk regulates region-specific neuroinflammation. Glia

Wu C, Chen J, Liu Y, Zhang J, Ding W, Wang S, Bao G, Xu G, Sun Y, Wang L, Chen L, Gu H, Cui B, Cui Z (2016) Upregulation of PSMB4 is associated with the necroptosis after spinal cord injury. Neurochem Res 41(11):3103-3112

Wu JR, Wang J, Zhou SK, Yang L, Yin JL, Cao JP, Cheng YB (2015) Necrostatin-1 protection of dopaminergic neurons. Neural Regen Res 10(7):1120-1124

Yamaguchi H, Maruyama T, Urade Y, Nagata S (2014) Immunosuppression via adenosine receptor activation by adenosine monophosphate released from apoptotic cells. Elife 3:e02172

Yang SH, Lee DK, Shin J, Lee S, Baek S, Kim J, Jung H, Hah JM, Kim Y (2017) Nec-1 alleviates cognitive impairment with reduction of Abeta and tau abnormalities in APP/PS1 mice. EMBO Mol Med 9(1):61-77

Yin X, Yin Y, Cao FL, Chen YF, Peng Y, Hou WG, Sun SK, Luo ZJ (2012) Tanshinone IIA attenuates the inflammatory response and apoptosis after traumatic injury of the spinal cord in adult rats. PLoS ONE 7(6):e38381

You Z, Savitz SI, Yang J, Degterev A, Yuan J, Cuny GD, Moskowitz MA, Whalen MJ (2008) Necrostatin-1 reduces histopathology and improves functional outcome after controlled cortical impact in mice. J Cereb Blood Flow Metab 28(9):1564-1573

Zhang N, Yin Y, Xu SJ, Wu YP, Chen WS (2012) Inflammation \& apoptosis in spinal cord injury. Indian J Med Res 135:287-296

Zhang S, Tang MB, Luo HY, Shi CH, Xu YM (2017) Necroptosis in neurodegenerative diseases: a potential therapeutic target. Cell Death Dis 8(6):e2905

Zhao H, Chen S, Gao K, Zhou Z, Wang C, Shen Z, Guo Y, Li Z, Wan Z, Liu C, Mei X (2017) Resveratrol protects against spinal cord injury by activating autophagy and inhibiting apoptosis mediated by the SIRT1/AMPK signaling pathway. Neuroscience 348:241-251

Zhou H, Chen L, Gao X, Luo B, Chen J (2012) Moderate traumatic brain injury triggers rapid necrotic death of immature neurons in the hippocampus. J Neuropathol Exp Neurol 71(4):348359

Zhu S, Zhang Y, Bai G, Li H (2011) Necrostatin-1 ameliorates symptoms in R6/2 transgenic mouse model of Huntington's disease. Cell Death Dis 2:e115 